\title{
Al Powered Unmanned Aerial Vehicle for Payload Transport Application
}

\author{
Reem Alshanbari \\ mmh Labs, Computer, Elecctrical and \\ Mathematical Sciences and \\ Engineering Division \\ King Abdullah University of Science \\ and Technology \\ Thuwal, Saudi Arabia \\ reem.alshanbari@kaust.edu.sa
}

\author{
Sherjeel Khan \\ mmh Labs, Computer, Elecctrical and \\ Mathematical Sciences and \\ Engineering Division \\ King Abdullah University of Science \\ and Technology \\ Thuwal, Saudi Arabia \\ sherjeel.khan@kaust.edu.s
}

$\underline{a}$

\author{
Muhammad Mustafa Hussain \\ mmh Labs, Computer, Elecctrical and \\ Mathematical Sciences and \\ Engineering Division \\ King Abdullah University of Science \\ and Technology \\ Thuwal, Saudi Arabia \\ Elecctrical Engineering and Computer \\ Science \\ University of California, Berkeley \\ California, USA \\ muhammad.hussain@kaust.edu.sa \\ mmhussain@berkeley.edu
}

\author{
Nazek El-Atab \\ mmh Labs, Computer, Elecctrical and \\ Mathematical Sciences and \\ Engineering Division \\ King Abdullah University of Science \\ and Technology \\ Thuwal, Saudi Arabia \\ nazek.elatab@kaust.edu.s
}

$\underline{a}$

\begin{abstract}
Recently unmanned aerial vehicles (UAV) have received a growing attention due to their wide range of applications. Here, we demonstrate UAVs with artificial intelligence (Al) capabilities for application in autonomous payload transport. An algorithm is developed for target detection with multiple phases on the ground, which once the target is detected, would trigger the release of the payload that is attached on the drone. The experimental results show that the average frame rate over $x$ seconds achieved a 19.4010717352 fps (frame per second) detection speed. Releasing the payload is achieved using a 3D printed system based on rack and pinion gears. In addition, auto flight program is developed to enable the autonomous movement of the drone. As a proof-of-concept, a small drone known as "Phantom DJI" is used for .6 kg autonomous payload transport along a predefined route to a target location.
\end{abstract}

Keywords - UAV; robotics; color detection; payload.

\section{INTRODUCTION}

Over the last decade, unmanned aerial vehicles (UAVs), commonly known as 'drones' have offered a diverse range of applications including environmental sensing, sampling, and surveillance [1]. UAVs are also considered as an important technology due to their ability to reach areas that human beings cannot easily access otherwise [2]. In order to enable the UAVs, and robotics in general, to work autonomously and assist people with daily tasks, they must be equipped with artificial intelligence ( $\mathrm{Al}$ ) algorithms that allows them to understand and communicate with the real world, as Ada Lovelace envisioned back in the early $19^{\text {th }}$ century [3]. For instance, image processing and computer vision-based $\mathrm{Al}$ algorithms have proven to be powerful tools for monitoring and detection applications [4]. In this paper, we report the design and development of an algorithm for target object identification based on color and edge detection. A small drone is then used to transport a payload to the target object/location. The proposed system allows the drones to detect objects, using multiple algorithms in order to improve its accuracy and release the payload in the correct location. To design the payload dropping system, we used polymer materials to $3 \mathrm{D}$ print a cost-effective and lightweight mechanical system based on rack and pinion gears. Finally, Software Development Kit (SDK) based program is used to enable the autonomous flight of the drone.

\section{UAV SYSTEM}

\section{A. System overview}

The system consists of the main UAV platform which is based on a Phantom 4 DJI drone, with external camera board Intel $^{\circledR}$ RealSense depth camera with powerful vision processor that uses 28 nanometer $(\mathrm{nm})$ process technology and supports up to $5 \mathrm{MIPI}$ camera serial interface 2 lanes to compute real-time depth images and accelerate output. We used Jetson Nano as a microcomputer as shown in Fig.1. with a quad-core 64-bit ARM CPU and a 128-core NVIDIA GPU. We installed on the microcomputer, JetPack 4.2 SDK which provides a complete desktop Linux environment. We used Open Source Computer Vision Library (OpenCV) which is free for both academic and commercial use. The algorithm developed by using Python 3 on the microcomputer for identifying color and detecting edges. Then, a program was developed to calculate and determine the centroid point in each shape. Moreover, a 
Pulse Width Modulation (PWM) servo driver board was added to the microcomputer over $12 \mathrm{C}$ to allow interfacing and controlling with Servo easier. The main platform is the Phantom $4 \mathrm{DJI}$. The selected camera and microcomputer are both powerful enough to work on visual computing applications. In order to deploy and release the payload, the UAV was programmed to fly on a predetermined path up and down in the field until it reaches the desired location.

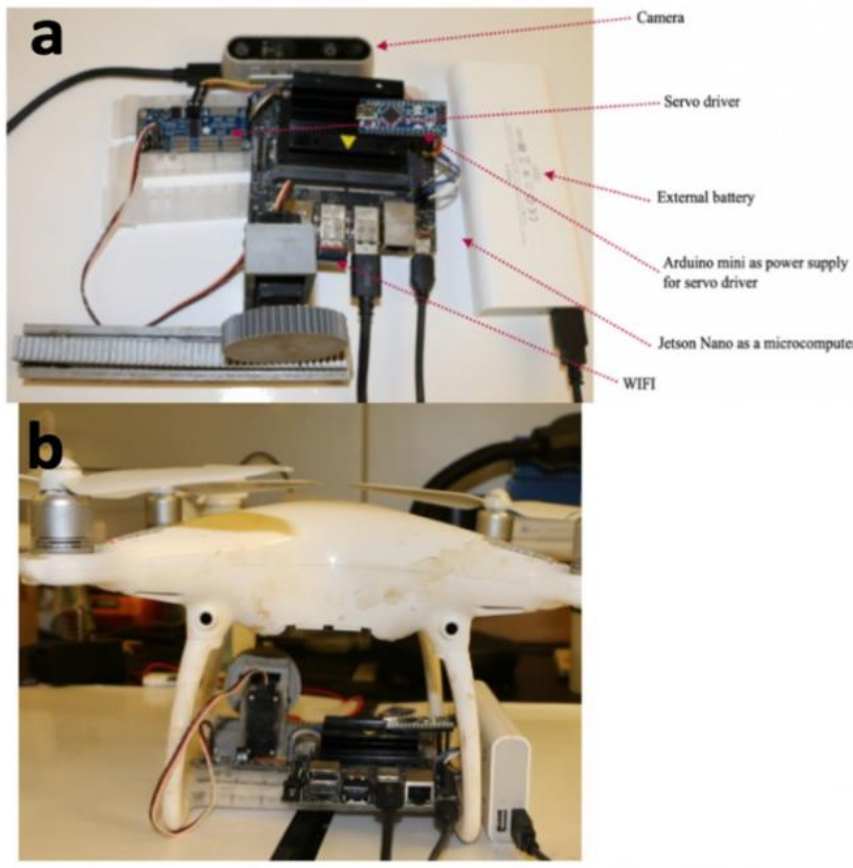

Fig. 1. (a) The components of the device. (b) The device attached to the drone

\section{B. Proposed Algorithm}

\section{First Phase:}

The initial phase of the algorithm works on detecting any frame that contains the color of the target object. It should be noted that all the different shadows use HSV color ranges as shown in Fig. 2. where the green color is presented as an example. The process of detecting multiple shades of each color and identifying them is noted in Fig. 3. this section is crucial since the color of the object might change during the time or under different environmental conditions. Another important aspect of the algorithm is the detection of the edges of the target object. This helps in reducing the error rate and therefore, the drone will be able to detect the target object more accurately.

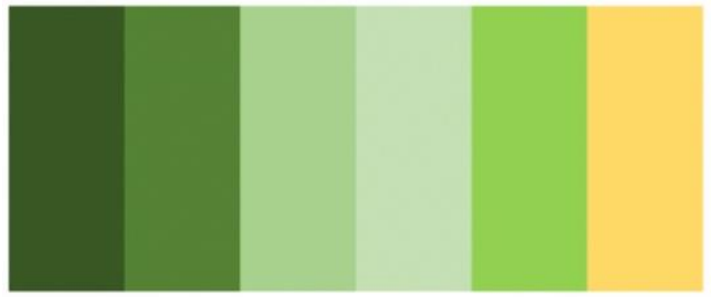

Fig. 2. Shades of green

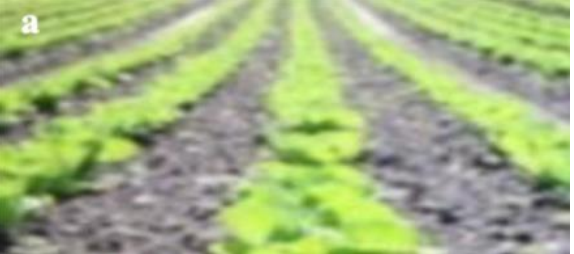

Original Image

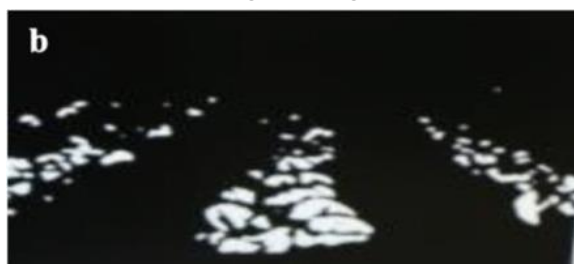

Binary Mask

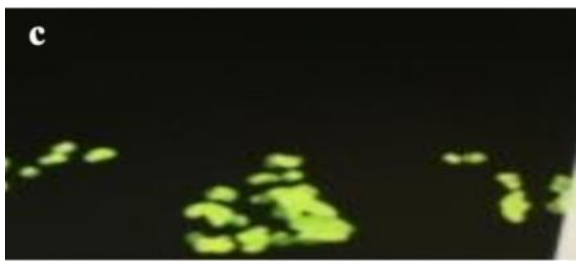

Output Result

Fig. 3. The process of color detection

\section{Second Phase:}

The second phase detects the edge of the object. Knowing the edges of the target object help in reducing the error when the drone releases the payload at the target location. In this phase, an edge detection operator and a multi- stage algorithm are used to detect a wide range of edges in these images as shown in Fig. 4 -5. The algorithm was originally developed by John F. Canny in 1986. Next, Hough lines algorithm is utilized to provide the precise object boundaries.

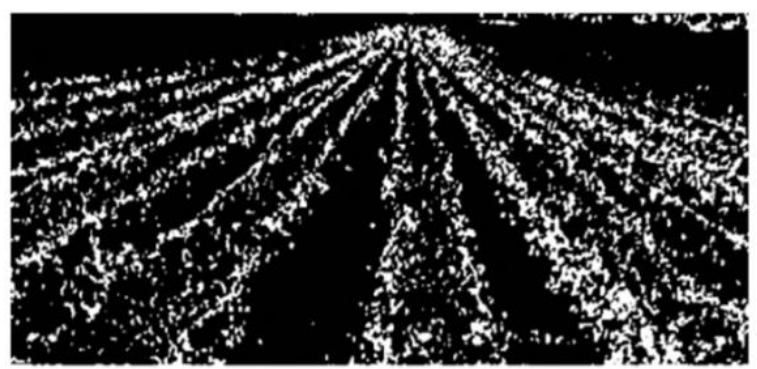

Fig. 4. Results after edge detection and Hough transformation of green object

The last step in this algorithm is object Realtime position which determining the centroid point of the detected shape that is made of pixels wherein, the centroid is simply the weighted average of all the pixels that constitute the shape resulting in the $x, y$ coordinates of the center. In this system, OpenCV is used to find the object center using moments, which consist of the weighted average of image pixel intensities converted to the binary system. Thus, the camera is attached on the drone such that it is facing the ground as shown in Fig.6. then, the coordinates of the center of the target object are calculated using the following equations: 


$$
\begin{aligned}
& C_{x}=\frac{M_{10}}{M_{00}} \\
& C_{y}=\frac{M_{01}}{M_{00}}
\end{aligned}
$$

where $C_{x}$ is the $x$ coordinate, $C_{y}$ is the $y$ coordinate of the centroid and $\mathrm{M}$ is the Moment [5].

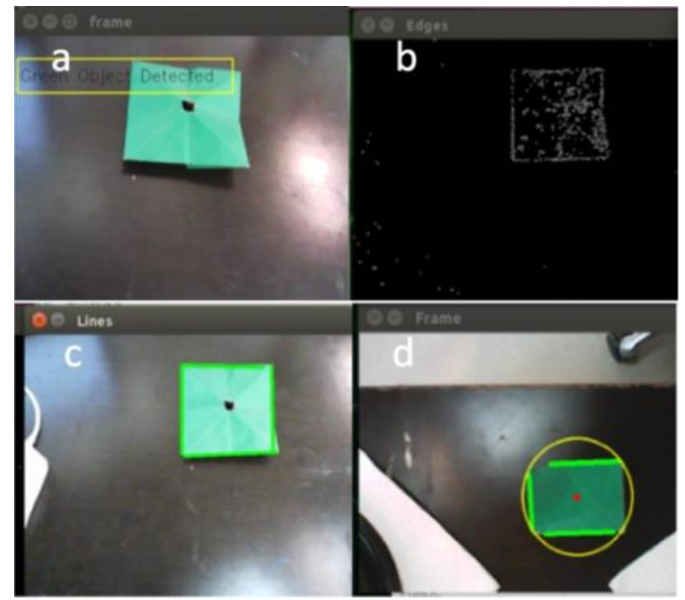

Fig. 5. Example of the algorithms applied on a green paper as an example. (a) Color detection, (b) edge-based, and (c) Hough transform and (d) Identify center point of the object

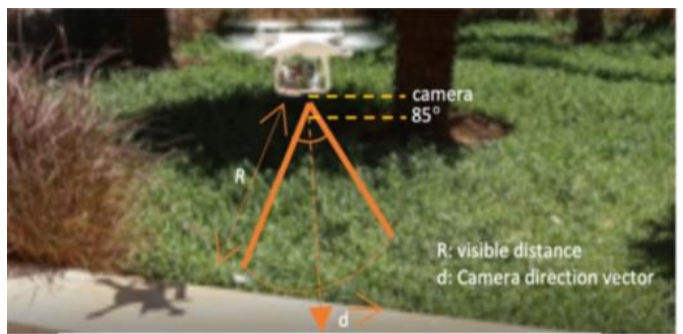

Fig. 6 . The camera attached to the drone

After performing the previously mentioned algorithms, the drone will be capable to make a decision. If the target location is accurately detected, the payload would be released, otherwise, the drone will keep moving on the predefined path until it finds the target with the smallest possible error rate.

\section{Autonomous mode}

Recently, it has been shown that critical aspects in UAV research field include localization, navigation, and mapping [6]. In this work, we previously developed a primary handler to enable an operator to control a UAV remotely. This enduser program allows the UAV to execute a self- flight task using Global Positioning System (GPS) information. We used a phone with an iOS operating system as the end-user program, which handles route planning. The end-user program was developed in the Objective-C programming language using Xcode (Apple Inc.) and the DJI mobile SDK [7]. The DJI SDK is geared toward developers who are interested in designing their DJI UAV software to be used along with the remote controller.
The iOS SDK connects to the UAV wirelessly through a remote controller. The mobile SDK then can connect to the remote controller with a USB cable [8]. We created a simple graphical user interface (GUI), as shown in Fig. 7. This GUI includes a display of GPS data with latitude. The custom GUI consists of an interface to create a navigational path for the UAV.

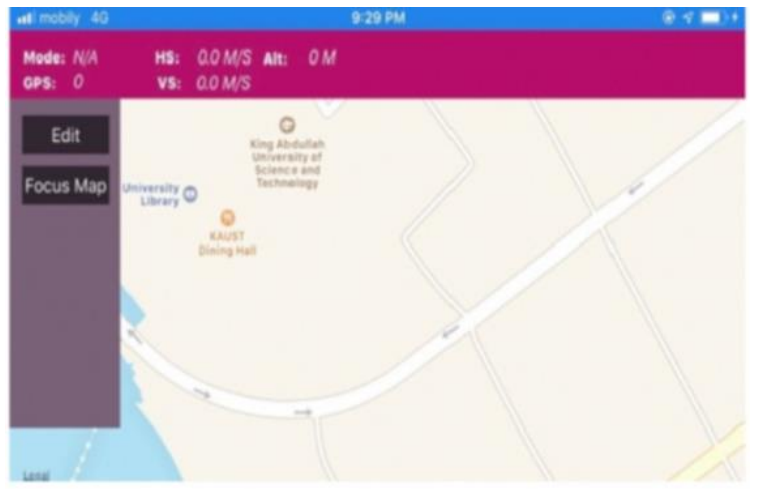

Fig. 7. The GUI of the system

\section{3D-PRINTED DROPPING MECHANIS}

Another challenge in this work is the design of a lightweight dropping system with a minimum number of electronic components in order to allow the drone to carry heavier payloads. Thus, a 'Rack and Pinion' slider is used to release the payload. The rack and pinion gear system is composed of two gears [9]. The usual round gear is called a "pinion" gear, and the straight gear is called a "Rack" gear as shown in Fig. 8. A rack and pinion system is a linear actuator that transforms rotational motion into linear movement [9]. The circular pinion joins the teeth on the linear "rack". Thus, when rotational motion is applied to the pinion, the rack moves linearly until it drops the payload.

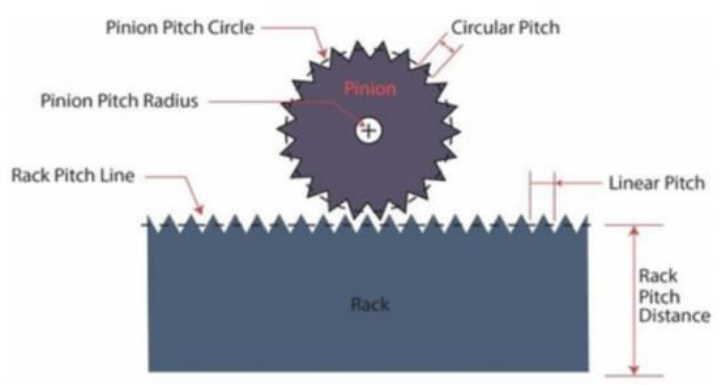

Fig. 8. Rack and pinion gear

In addition, sliders are designed in which the rack is placed as shown in Fig. 9. The payload would be placed inside the slider as well. Thus, when the rack moved linearly, it comes in contact with the payload and causes its release from the dropping system. The release system is designed using the solid works software and 3D printed using the PLA polymeric material (polylactic acid or polylactide) which is both a low cost and effective material. The movements of the rack and pinion shown in Fig. 10. 


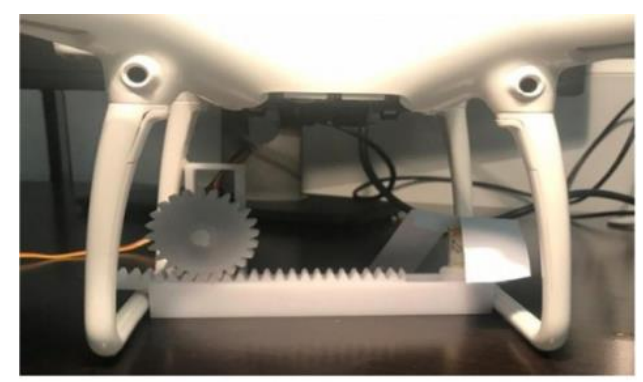

Fig. 9. The 3D printed dropping system
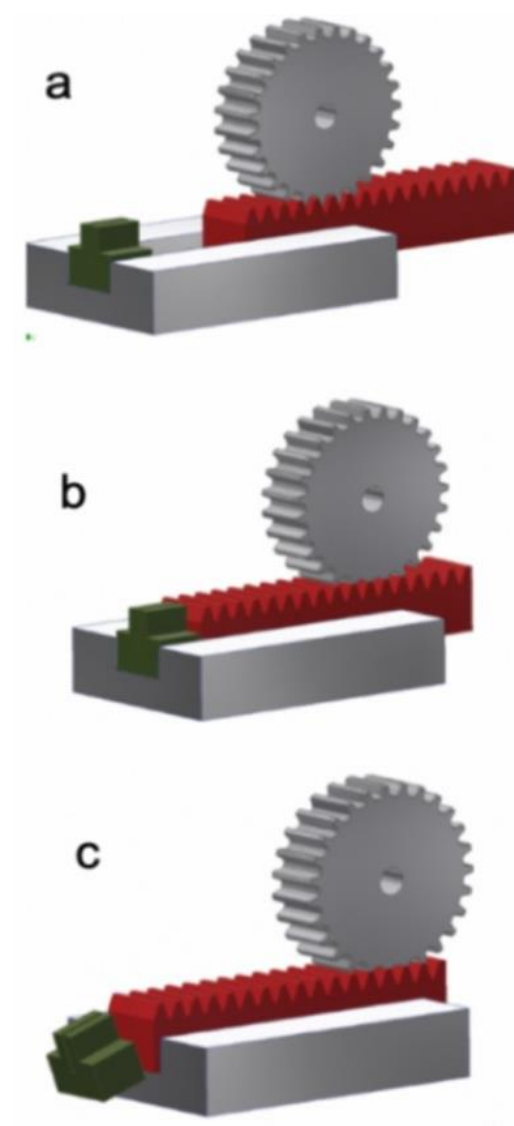

Fig. 10. Movements of the 'Rack and Pinion

\section{EXPERIMNTAL AMD RESULTS}

The experiment was conducted on May 5, 2019, at 10:15 am in sunny and windy weather. The test flight occurred at King Abdullah University of Science and Technology (KAUST), Thuwal, SA. As the first step, we manually collected static locations for all points over which the drone would hover by using an external GPS as shown in Fig. 11. This external GPS can perform a maximum of 10 position updates per second for high-speed, high-sensitivity logging or monitoring. Whereas the phone's GPS would provide an accuracy of around 5-8 meters, but by using the external GPS we can achieve an accuracy of within 3 meters.
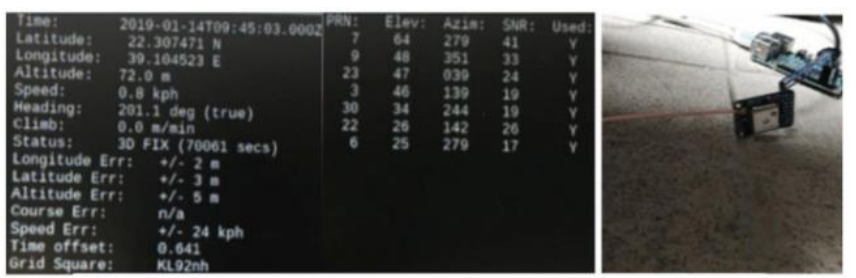

Fig. 11. GPS unit integrated with microcomputer

We took this GPS information to feed the latitude and longitude of the different positions into the end-user program the camera was attached on the UAV and faced towards the ground. The path of the flight test shown in Fig. 12. The drone was able to fly on the predetermined path with 1- 3 meters errors. The response time of the drone system to detect the target object and release the payload has also been tested at different altitudes and in different environmental conditions (indoors and outdoors). It is found that as the drone is flying at a higher altitude, the response time increases. For instance, at a height of $2 \mathrm{~m}$, the drone took $2 \mathrm{~s}$ to detect the target and make a decision to release the payload while at a height of $6 \mathrm{~m}$, the response time increases to $5 \mathrm{~s}$. The test showed consistent results where it was conducted indoors and outdoors, which shows the ability of the Al powered drone to make accurate decisions regardless of the outer environment conditions. However, the GPS and auto mode functionalities are not supported indoors, thus, the flight has been controlled. In the outdoor test, after determining the series of GPS coordinates, we executed the algorithm to guide the aircraft along an assigned path defined by the series of waypoints. The path consisted of 10 points in the form of a curved line, each of which had a specific identifier and was located in a different position. We then pressed "start the flight" on the end-user program. The UAV operator did not touch the remote controller or intervene in any way until the drone landed 3 minutes later.

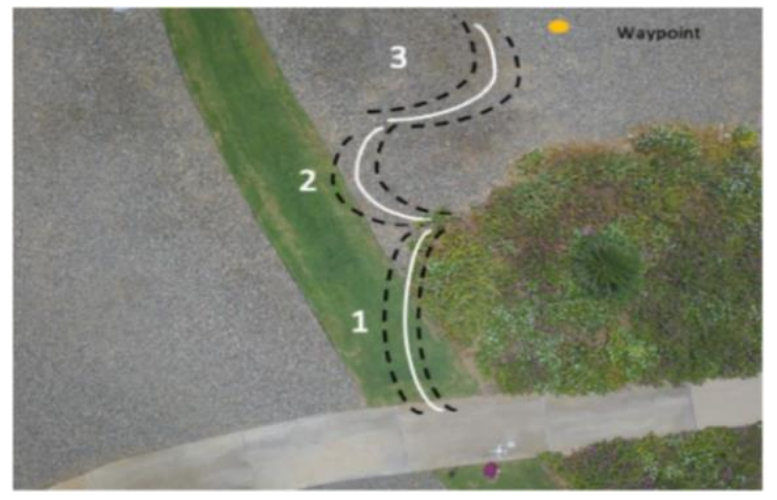

Fig. 12. The location of the flight test

\section{A. Accuracy of the Navigation}

To test the flight navigation accuracy, three flights were performed using the same series of GPS points, the tests were conducted at different times. A comparison of the three flights showed that the drone could fly precisely according to the predetermined GPS points with an error of $\leq 3$ meters. Three pictures selected on the same GPS points and the same ground area as shown in Fig. 13. 

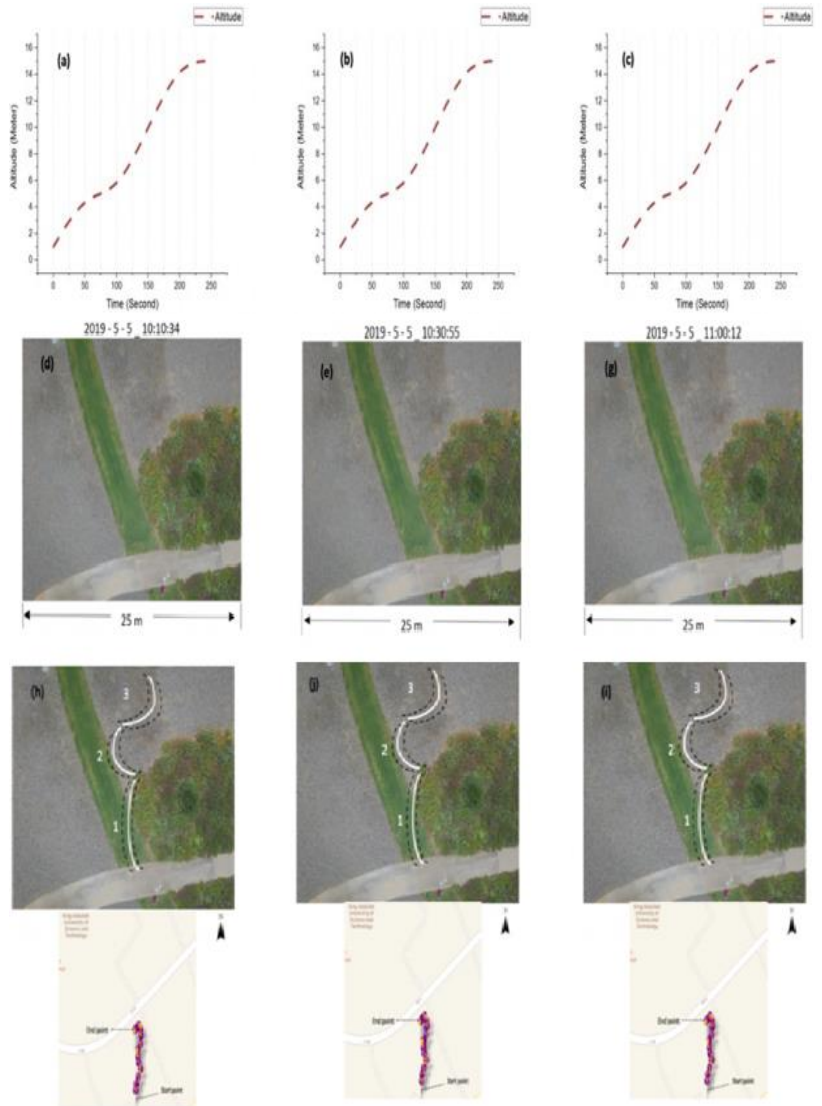

Fig. 13. Comparison of three flights with same altitude (c,b and g) along with the same set of waypoints; and three aerial photographs over the the waypoints ( $d$,e and g). The path of flight represented ( $h, j$ and i) describe the location of aerial photographs and waypoints, respectively. Purple points represent the path of the auto flight

\section{CONCLUSION}

In this work, we demonstrated the use of drones in the payload transport application. The drone was equipped with Al capability to detect the color and edge of the target object/target location for payload release. The use of multiple algorithms to detect both the color and edge of the object helps in reducing the error rate. We show that the Phantom drone is capable of transporting up to $.6 \mathrm{~kg}$ payloads. The release mechanism is achieved using a 3D printed rack and pinion gears. In addition, the drone is equipped with the capability to fly autonomously using the SDK resources. The work presented here is a proof-ofconcept for small drones' application in payload transport and thus similar algorithms can be applied on bigger drones with improved performance payload transport and deployment

\section{ACKNOWLEDGMENT}

The work is supported by the King Abdullah University of Science and Technology (KAUST) Office of Sponsored Research (OSR) under Award No. Sensor Innovation Initiative OSR-2015-Sensors-2707 and KAUST-KFUPM Special Initiative OSR-2016-KKI-2880.

\section{REFERENCES}

[1] Sareh, Pooya \& Chermprayong, Pisak \& Emmanuelli, Marc \& Nadeem, Haris \& Kovac, Mirko. (2018). Rotorigami: A rotary origami protective system for robotic rotorcraft. Science Robotics. 3. eaah5228. 10.1126/scirobotics.aah5228.

[2] Soleimani, Amir \& Nasrabadi, N.M. \& Griffith, Elias \& Ralph, Jason \& Maskell, Simon. (2018). Convolutional Neural Networks for Aerial Vehicle Detection and Recognition. 186-191. 10.1109/NAECON.2018.8556672

[3] Clabaugh, Caitlyn \& Matarić, Maja. (2018). Robots for the people, by the people: Personalizing human-machine interaction. Science Robotics. 3. eaat7451. 10.1126/scirobotics.aat7451

[4] A. Ruangwiset, "The application of unmanned aerial vehicle to precision agriculture: Verification experiments of the power consumption," 2014 International Conference on Information Science, Electronics and Electrical Engineering, Sapporo, 2014, pp. 968-971.

[5] OpenCv.(2015):

https://docs.opencv.org/3.1.0/dd/d49/tutorial_py_contour_features.html

[6] P. Tripicchio, M. Satler, G. Dabisias, E. Ruffaldi and C. A. Avizzano, "Towards Smart Farming and Sustainable Agriculture with Drones," 2015 International Conference on Intelligent Environments, Prague, 2015, pp. 140-143. doi: 10.1109/IE.2015.29

[7] Developer.dji.com. (2019). DJIWaypointMission. [online] Available at: https://developer.dji.com/iframe/mobile-sdkdoc/

android/reference/dji/sdk/MissionManager/DJIWaypointMission.html\#isPa usable() [Accessed

10 May 2019].

[8] Zeming Lu, Fusaomi Nagata, Keigo Watanabe, and Maki K. Habib.

(2017). iOS application for

quadrotor remote control. Artif. Life Robot. 22, 3 (September 2017), 374379. DOI:

[9] Klocke F. (2014) Gear Cutting. In: The International Academy for Production Engineering, Laperrière L., Reinhart G. (eds) CIRP Encyclopedia of Production Engineering. Springer, Berlin, Heidelberg 